\title{
Why surgery won the SYNTAX trial and why it matters
}

Michael Mack, MD, Heike Baumgarten, MD, and Bruce Lytle, MD

\begin{abstract}
The Synergy Between Percutaneous Coronary Intervention With Taxus and Cardiac Surgery (SYNTAX) trial cause of death analysis shows that cardiac death due to myocardial infarction is 10 times higher with percutaneous coronary intervention than coronary artery bypass grafting in the higher-risk patients. There was a clear advantage for surgery in the prevention of death in both the intermediate and high SYNTAX score groups with 3-vessel disease and in the high SYNTAX score group with left main disease, and that incremental advantage is statistically significant and widening with time, which should be transparently communicated to patients. (J Thorac Cardiovasc Surg 2016;152:1237-40)
\end{abstract}

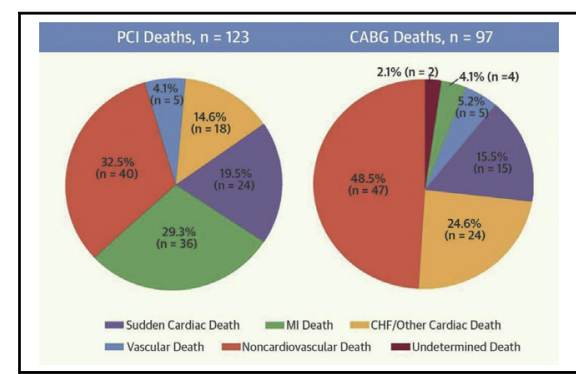

Causes of deaths in the SYNTAX randomized cohort: $\mathrm{PCl}$ and $\mathrm{CABG}$ comparison.

\begin{abstract}
Central Message
The SYNTAX trial cause of death analysis shows that cardiac death due to MI is 10 times higher with PCI than CABG in the higher-risk patients, which should be transparently communicated to patients.
Perspective
The SYNTAX trial showed the benefit of CABG over PCI by 5 years in patients with multivessel and left main coronary artery dis- ease. This cause of death analysis adds insight as to why CABG is beneficial. The study re- veals that cardiac death due to $\mathrm{MI}$ is 10 times higher with PCI than CABG in the higher-risk patients. This important finding should be transparently communicated to patients.

See Editorial Commentary page 1241.

See Editorial page 1227.
The results of the Synergy Between Percutaneous Coronary Intervention With Taxus and Cardiac Surgery (SYNTAX) trial (NCT00114972) have become widely disseminated over the past 7 years. The trial's primary 1-year end point results and the 5-year outcomes have been published, as have numerous prespecified subgroup analyses and post hoc exploratory studies. ${ }^{1-4}$ The results have consistently demonstrated the benefit of coronary artery bypass grafting $(\mathrm{CABG})$ compared with percutaneous coronary

From the Heart Hospital Baylor Plano, Plano, Tex.

Received for publication April 25, 2016; accepted for publication April 26, 2016; available ahead of print June 7, 2016.

Address for reprints: Michael Mack, MD, Heart Hospital Baylor Plano, 1100 Allied

Dr, Plano, TX 75075 (E-mail: Michael.mack@bswhealth.org). $0022-5223 / \$ 36.00$

Copyright (C) 2016 by The American Association for Thoracic Surgery http://dx.doi.org/10.1016/j.jtcvs.2016.04.083 intervention (PCI), especially in those with advanced coronary artery disease (CAD) or diabetes. A new analysis of the SYNTAX trial recently has been performed that offers intriguing new insights into the trial that help explain these outcomes and why there is an advantage for CABG over PCI in patients with advanced CAD. ${ }^{5}$

Let us first summarize the overall SYNTAX trial design and outcomes, and then discuss the important findings of this new study. The SYNTAX trial was a prospective, multinational, randomized trial conducted in 85 centers in 17 countries in the United States and Europe between 2005 and 2007. In this study, 1800 patients with de novo left main or 3-vessel disease were randomly assigned to undergo CABG or PCI with first-generation paclitaxel-eluting stents (Taxus Express, Boston Scientific, Natick, Mass). In addition, there were 2 nested registries of patients who qualified 


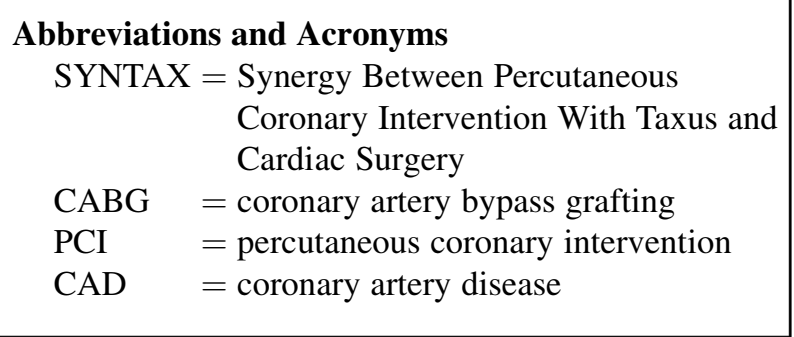

for only 1 of the 2 randomized treatments, a PCI Registry usually for patient-related factors $(\mathrm{n}=198)$ and a CABG registry mainly for coronary anatomic issues $(n=1077)$, thus creating an "all-comer" trial of 3075 patients.

One of the unique aspects of this trial is that patients were grouped on the basis of not only the number of coronary vessels that were significantly stenotic but also by a grading system, the SYNTAX score, reflecting the complexity of the $\mathrm{CAD}$, including the diffuseness of the disease and the extent of the involvement of disease in the vessels regardless of whether those stenoses were judged as significantly narrowed. On the basis of this scoring of the complexity of the CAD, patients were divided into tertiles: low complexity (0-22), intermediate complexity (23-32), and highly complex $(>32)$ disease.

The primary end point of the trial was a composite of major adverse cardiac or cerebrovascular events composed of all-cause mortality, stroke, myocardial infarction (MI), and need for repeat revascularization at 1 year, with all patients followed for at least 5 years.

The primary end point analysis demonstrated that "CABG remains the standard of care for patients with three-vessel or left main coronary artery disease, since the use of CABG, as compared with PCI, resulted in lower rates of the combined end point of major adverse cardiac or cerebrovascular events at 1 year." ${ }^{1}$ However, the main reason for the benefit of CABG at 1 year mainly was due to the increased need for repeat revascularization with PCI. Subsequent analysis at 5 years confirmed that "CABG should remain the standard of care for patients with complex lesions (high or intermediate SYNTAX scores). For patients with less complex disease (low SYNTAX scores) or left main coronary disease (low or intermediate SYNTAX scores), PCI is an acceptable alternative." 2 The 5-year analysis also found that although the superiority of CABG at 1 year mainly was due to the need for repeat revascularization with PCI, there was a now a significant advantage to CABG not only in the need for repeat revascularization but also in the "hard components of the composite end point" of death, stroke, and MI. In fact, there was a significant survival advantage for CABG in the patients with intermediate and high SYNTAX scores, with the death rate of CABG versus PCI being $9.6 \%$ versus $16.3 \%(P=.047)$ and $8.8 \%$ versus $17.8 \%(P=.02)$, respectively.
There are a number of important takeaways from this trial. The incremental benefit of surgery is greater the higher the SYNTAX score, that is, the worse and more diffuse the coronary disease. For patients in the lowest SYNTAX score, the group advantages of surgery versus PCI were not statistically significant; however, the trends are clear, and the differences are continuing to widen with the passage of time. For patients in the intermediate and highest SYNTAX groups, the incremental advantage of surgery is highly statistically significant, and these differences are continuing to widen at this time. Although complexity of disease as measured by the SYNTAX score matters for PCI, it does not for CABG.

Shedding further insight into the SYNTAX trial outcomes is a detailed post hoc analysis examining the causes of death in the trial and adds additional important insights as to the reasons for the advantages of CABG. ${ }^{5}$ The study was an extensive, detailed, blinded analysis of the causes of death in all 3075 trial and registry patients. So why is this particular study important and what can we learn from the treatment of these patients a decade ago? Moreover, how and why is this analysis relevant to current clinical practice?

The study by Milojevic and colleagues ${ }^{5}$ analyzed the causes of death in both PCI and CABG cohorts and found that there were $97(11.4 \%)$ all-cause deaths in patients undergoing CABG and $123(13.9 \%)$ all-cause deaths in patients undergoing PCI at 5 years. However, there was a 10-fold higher rate of cardiac death due to MI in PCItreated patients compared with CABG-treated patients $(4.1 \%$ vs $0.4 \%, P<.0001)$. This was particularly striking in patients with 3-vessel disease, high SYNTAX scores, or diabetes. Patients who died had a higher risk profile at baseline than those who were still alive after 5 years in both cohorts. PCI alone was not an independent predictor of all-cause mortality, but it was an independent predictor of cardiac death (hazard ratio, $1.55 ; 95 \%$ confidence interval, $1-2.33 ; P=.045)$. Of note, procedural events including incomplete revascularization were associated with an increased risk of cardiac death (hazard ratio, 1.89; $95 \%$ confidence interval, 1.20-2.98; $P=.006$ ). In the CABG group, chronic obstructive pulmonary disease and renal failure were the only independent predictors for cardiac death.

In this cause of death analysis, there was a clear advantage for surgery in the prevention of death in both the intermediate and high SYNTAX score groups with 3-vessel disease and in the high SYNTAX score group with left main disease, and that incremental advantage is statistically significant and widening with time. In discussions of these issues over the last 30 years, some have tried to make the point that reintervention is a "nonevent." That is not true of death.

These observations make sense and fit well with the consistent demonstration of the advantages of surgery for patients with triple-vessel disease. The advantages have been most striking for patients with intermediate or high 
SYNTAX scores, but seem to exist for patients with low scores as well, although requiring more follow-up.

For other subgroups, particularly those with left main stenosis and less diffuse distal disease and lower SYNTAX scores, the situation is less clear. Patients with left main stenosis combined with low or intermediate SYNTAX scores had equivalent 5-year major adverse cardiac or cerebrovascular events and survival regardless of treatment modality, and it may be that PCI is an effective treatment option for those subgroups. Trials of that hypothesis in low and intermediate SYNTAX score left main disease (Evaluation of the Xience Everolimus Eluting Stent Versus Coronary Artery Bypass Surgery for Effectiveness of Left Main Revascularization [EXCEL] trial; NCT01205776) are under way. For patients with complex left main stenosis, triple-vessel disease, and high Syntax scores, surgery is superior.

These findings are not startling and have shown that patients with isolated triple-vessel disease experience fewer cardiac events when treated with surgery. Those with intermediate or high SYNTAX scores are less likely to die when treated with surgery. The study by Milojevic and colleagues ${ }^{5}$ has given us some insight into why this is true.

Another possible reason for the better outcomes with CABG in the SYNTAX trial was the higher rate of complete revascularization There was incomplete revascularization in $43.3 \%$ of patients in the PCI arm and in $36.8 \%$ of patients in the CABG arm. Incomplete revascularization was associated with increased risk for major adverse cardiac or cerebrovascular events 3 years after PCI, but incomplete revascularization in the $\mathrm{CABG}$ group could not be identified as a predictor for worse outcomes. Another interesting post hoc analysis is the quantification of the degree of incomplete revascularization after PCI by calculation of a residual SYNTAX score. ${ }^{6}$ The residual SYNTAX score is useful to quantify and risk-stratify the degree and complexity of residual stenosis after PCI. Specifically, a residual SYNTAX score greater than 8.0 after PCI in patients with moderateand high-risk coronary disease is associated with a poor 30-day and 1-year prognosis. The clinical implications of this are clear: If there is consideration of PCI for patientrelated reasons, consideration of the amount of residual untreated disease that will remain after PCI is important because that adversely affects prognosis.

An editorial by Boden and Mancini, ${ }^{7}$ which accompanies the article by Milojevic and colleagues ${ }^{5}$ in the Journal of the American College of Cardiology, emphasizes a number of additional important points. First, patients receiving optimal medical therapy, defined as the combination of at least 1 antiplatelet drug, beta-blocker, statin, and angiotensin-converting enzyme inhibitor, have significantly lower rates of death after both CABG and PCI. In fact, it has been recently demonstrated that optimal medical therapy was underused in the SYNTAX CABG group, despite an association between optimal medical therapy and a significant reduction in mortality 5 years after CABG. ${ }^{8}$ It is recommended that these findings should promote universal adoption of guideline-directed optimal medical therapy for the secondary prevention of cardiovascular events in patients undergoing any revascularization.

Boden and Mancini ${ }^{7}$ also state that many cardiologists and internists continue to recommend PCI to patients with complex, multivessel CAD despite the data from SYNTAX and other randomized trials. Evidence of this comes from New York State data, which found that only $53 \%$ of patients with an indication for CABG are recommended for surgery if the treatment decision is left to an interventional cardiologist alone. ${ }^{?}$

An additional critique of the SYNTAX trial is that it is now dated and not relevant to current practice because of the use of first-generation drug-eluting stents. It has been proffered that the use of the newer-generation drug-eluting stents may have yielded different results than those of the SYNTAX trial, However, that is not the case. In the Bypass Surgery Versus Everolimus-Eluting Stent Implantation for Multivessel Coronary Artery Disease trial, which enrolled 880 patients, the primary composite end point (death, MI, and target vessel revascularization at a median follow-up of 4.6 years) occurred more frequently in the PCI group (CABG $10.6 \%$ vs PCI $15.3 \%$, $P=.004){ }^{10}$

Surgery may have won the SYNTAX trial because of better long-term results of $\mathrm{CABG}$ in patients with complex $\mathrm{CAD}$, but CABG can and needs to be improved by the increased use of arterial revascularization, taking appropriate measures to minimize stroke and ensuring adherence to guideline-directed medical therapy postoperatively.

In the end, treatment decisions are up to the patient, but informed consent concerning the treatment options for patients with coronary disease should include a discussion of these data, particularly in regard to survival. Furthermore, physician therapeutic recommendations must be based on awareness of these data. At present, there are data seeming to indicate that the majority of patients with 3 -vessel disease receiving invasive treatment in the United States are being treated with PCI, an outcome that is not congruent with these outcome findings.

The overall results and multiple subanalyses of the SYNTAX trial demonstrate that CABG is the preferred approach for coronary revascularization in patients with intermediate and advanced degrees of complexity of multivessel CAD. The recent cause of death analysis shows that one of the major advantages of CABG is a 10-fold less incidence of cardiac death due to MI at 5 years (Figure 1). Other analyses of the trial show that completeness of revascularization matters and that if PCI is a consideration, a calculation of remaining untreated CAD should be performed and those patients who are likely to have a high residual SYNTAX score should be strongly considered for CABG. Optimal 


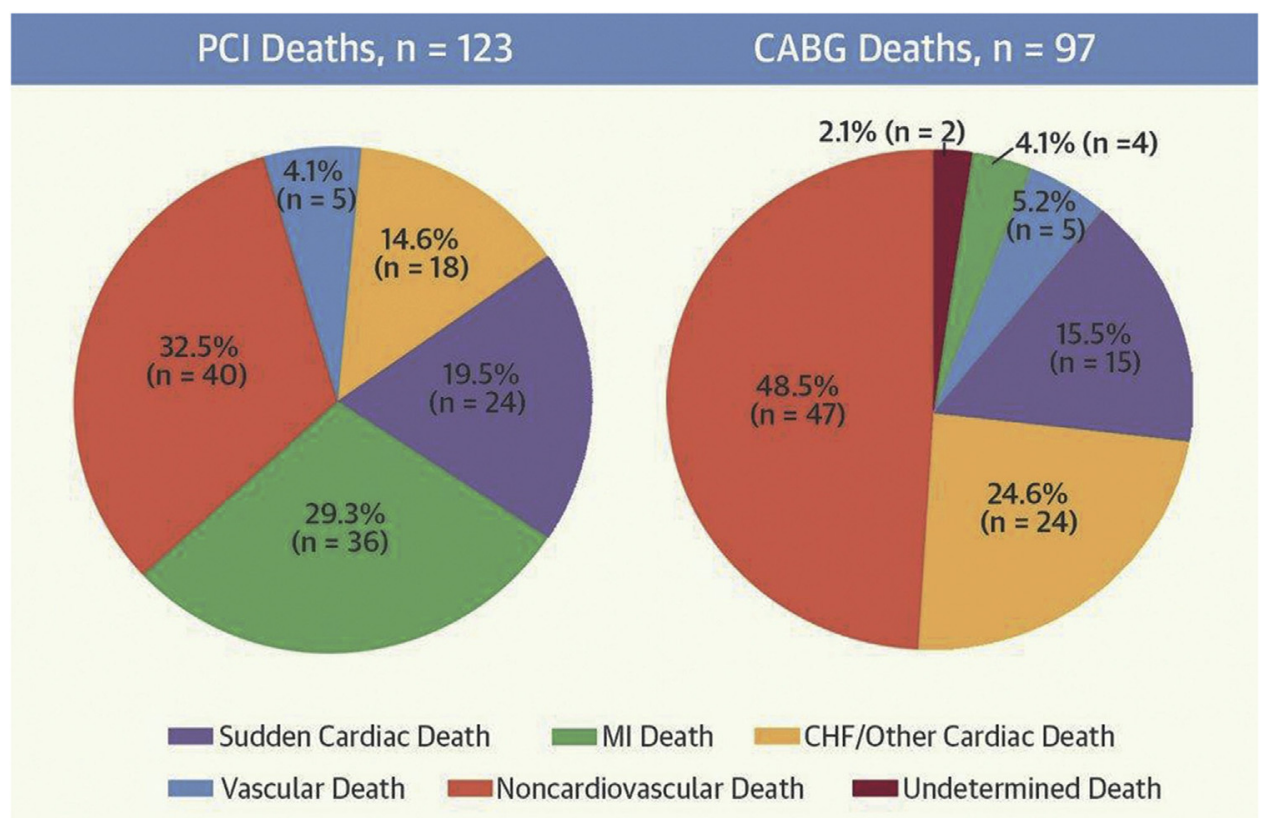

FIGURE 1. Causes of deaths in the SYNTAX randomized cohort: Percutaneous coronary intervention $(P C I)$ and coronary artery bypass grafting $(C A B G)$ comparison. $M I$, Myocardial infarction; $C H F$, congestive heart failure.

medical therapy has been demonstrated to improve outcomes after both CABG and PCI, and intensive efforts should be focused adherence to this treatment. Finally, although there is a strong patient preference for a lessinvasive treatment of their $\mathrm{CAD}$, patients with advanced disease should be made aware that the choice of PCI puts them at a increased risk of death relative to CABG, and a full and transparent discussion should occur regarding the implications of their decision.

\section{Conflict of Interest Statement}

Authors have nothing to disclose with regard to commercial support.

\section{References}

1. Serruys PW, Morice MC, Kappetein AP, Colombo A, Holmes DR, Mack MJ, et al. Percutaneous coronary intervention versus coronary-artery bypass grafting for severe coronary artery disease. N Engl J Med. 2009; 360:961-72.

2. Mohr FW, Morice MC, Kappetein AP, Feldman TE, Ståhle E, Colombo A, et al. Coronary artery bypass graft surgery versus percutaneous coronary intervention in patients with three-vessel disease and left main coronary disease: 5-year follow-up of the randomised, clinical SYNTAX trial. Lancet. 2013;381:629-38.

3. Head SJ, Davierwala PM, Serruys PW, Redwood SR, Colombo A, Mack MJ, et al. Coronary artery bypass grafting vs. percutaneous coronary intervention for patients with three-vessel disease: final five-year follow-up of the SYNTAX trial. Eur Heart J. 2014;35:2821-30.
4. Head SJ, Mack MJ, Holmes DR Jr, Mohr FW, Morice MC, Serruys PW, et al. Incidence, predictors and outcomes of incomplete revascularization after percutaneous coronary intervention and coronary artery bypass grafting: a subgroup analysis of 3-year SYNTAX data. Eur J Cardiothorac Surg. 2012;41:535-41.

5. Milojevic M, Head SJ, Parasca CA, Serruys PW, Mohr FW, Morice MC, et al. Causes of death following PCI versus CABG in complex CAD: 5-year followup of SYNTAX. J Am Coll Cardiol. 2016;67:42-55.

6. Généreux P, Palmerini T, Caixeta A. Quantification and impact of untreated coronary artery disease after percutaneous coronary intervention. The residual SYNTAX (Synergy Between PCI With Taxus and Cardiac Surgery) Score. J Am Coll Cardiol. 2012;59:2165-74.

7. Boden WE, Mancini GB. CABG for complex CAD: when will evidence-based practice align with evidence-based medicine? J Am Coll Cardiol. 2016;67:56-8.

8. Iqbal J, Zhang YJ, Holmes DR, Morice MC, Mack MJ, Kappetein AP, et al. Optimal medical therapy improves clinical outcomes in patients undergoing revascularization with percutaneous coronary intervention or coronary artery bypass grafting: insights from the Synergy Between Percutaneous Coronary Intervention with TAXUS and Cardiac Surgery (SYNTAX) trial at the 5-year follow-up. Circulation. 2015;131:1269-77.

9. Hannan EL, Racz MJ, Gold J, Cozzens K, Stamato NJ, Powell T, et al. American College of Cardiology and American Heart Association. Adherence of catheterization laboratory cardiologists to American College of Cardiology/American Heart Association guidelines for percutaneous coronary interventions and coronary artery bypass graft surgery: what happens in actual practice? Circulation. 2010;121:267-75.

10. Park SJ, Ahn JM, Kim YH, Park DW, Yun SC, Lee JY, et al. Trial of everolimuseluting stents or bypass surgery for coronary disease. N Engl J Med. 2015;372: 1204-12.

Key Words: coronary artery disease, coronary artery bypass grafting, percutaneous coronary intervention 